\title{
An efficient wavefield inversion for transversely isotropic media with a vertical axis of symmetry
}

\author{
Chao Song, Tariq Alkhalifah \\ King Abdullah University of Science and Technology (KAUST), \\ Physical Sciences and Engineering Division, \\ Thuwal, Saudi Arabia, 23955 \\ (January 27, 2020) \\ Running head: Acoustic VTI inversion
}

\begin{abstract}
Conventional full waveform inversion (FWI) aims at retrieving a high-resolution velocity model directly from the wavefields measured at the sensor locations resulting in a highly nonlinear optimization problem. Due to the high nonlinearity of FWI (manifested in one form in the cycle-skipping problem), it is easy to fall into local minima. Considering that the Earth is truly anisotropic, a multi-parameter inversion imposes additional challenges in exacerbating the null-space problem and the parameter tradeoff issue. We formulate an optimization problem to reconstruct the wavefield in an efficient matter with background models by using an enhanced source function (which includes secondary sources) in combination with fitting the data. In this two-term optimization problem to fit the wavefield to the data and to the background wave equation, the inversion for the wavefield is linear. As we keep the modeling operator stationary within each frequency, we only need one matrix inversion per frequency. The inversion for the anisotropic parameters are handled in a separate optimization using the wavefield and the enhanced source function. As velocity is the dominant parameter controlling the wave propagation, it is updated first. Thus, this
\end{abstract}


reduces undesired updates for anisotropic parameters due to the velocity update leakage. We show the effectiveness of this approach in reducing parameter tradeoff with a distinct Gaussian anomaly model. We find that in using the parameterization $v_{h}, \eta, \epsilon$ to describe the VTI model in the inversion, we end up with high resolution and minimal tradeoff compared to conventional parameterizations for the anisotropic Marmousi model. Application on $2 \mathrm{D}$ real data also shows the validity of the proposed method. 


\section{INTRODUCTION}

Conventional FWI aims at minimizing the misfit between observed and predicted data extracted from the wavefields at the sensor locations (Tarantola, 1984; Virieux and Operto, 2009). After decades spent in the development of the FWI theory and the enhanced computer capabilities, FWI has become a practical and an effective tool to estimate highresolution medium parameters especially when a sufficiently accurate initial model is provided. However, the objective function based on the $l_{2}$ norm least-squares measurement is not convex as a function of the model. As a result, it is easy for FWI to fall into a local minimum model when we use gradient-based optimizing methods, especially when low-frequency components of the data are missing. The success of conventional FWI depends on the availability of low-frequency information as well as large offsets in the data to initially reconstruct the low-wavenumber part of the model. New formulations aiming at mitigate the cycle-skipping problem have been proposed (Leeuwen and Herrmann, 2013; Alkhalifah and Wu, 2014, 2016). Unlike conventional FWI based on minimizing the difference between predicted and observed data in a least-square sense subject to the predicted data satisfying the wave equation, wavefield reconstruction inversion (WRI) uses the wave equation as a regularization term in inverting for the wavefield. This optimization enlarges the search space by inverting for the wavefield and the model as independent parameters (Leeuwen and Herrmann, 2013, 2015). By relaxing the modeling accuracy and extending the source beyond a point source, a wavefield can be reconstructed to fit the data (Abubakar et al., 2009; Wang et al., 2016, 2017). In these approaches, they require many relatively expensive iterations, as the model is updated at every iteration. In order to improve the computational efficiency, we use a modification to WRI (Leeuwen and Herrmann, 2013), that we refer to as efficient wavefield inversion (EWI), to calculate medium parameter per- 
turbations using a direct division (deconvolution) method (Alkhalifah, 2019; Alkhalifah and Song, 2019).

However, using simulated data corresponding to an isotropic assumption of the Earth yields poor results in many areas as it ignores the anisotropic reality of the true Earth. As a result, multi-parameter inversion was proposed to provide better data fitting. There are multiple parameters governing wave propagation in anisotropic media, and often data sensitivity to these parameters is not uniform. So parameter update leakage may cause inaccurate inversion results (Alkhalifah and Guitton, 2016). For transversely isotropic media with a vertical axis of symmetry (VTI), the analysis of the FWI dependency on the anisotropy parameters is crucial for understanding the parameter tradeoff and finding an optimal parametrization (Alkhalifah and Plessix, 2014). Under the first-order Born approximation, which dominates the FWI update, the parameter perturbation radiation patterns highlight the angles of data sensitivity from the model point prospective. Over the past few years, the VTI medium parameterization and the corresponding multi-parameter waveform inversion have been studied. For the conventional surface seismic setup, Alkhalifah (2016) argues that a parametrization made up of the horizontal velocity $v_{h}$, and the anisotropic parameters $\eta$ and $\epsilon$ provide optimal features for multi-parameter FWI in VTI media with minimal tradeoff. In this parameterization, the horizontal velocity is first reconstructed at the early stage of FWI using the diving waves without minimal tradeoff influence from other anisotropic parameters. Thus, $\epsilon$ information is extracted from reflected waves at small scattering angles, while $\eta$ is sensitive to the mid-range scattering angles, and expected to be retrieved prior to FWI from, for example, tomography. These features have been demonstrated in acoustic VTI media inversion using a scattering integral method (Djebbi et al., 2017), and in elastic VTI media using conventional elastic FWI (Guitton and Alkhalifah, 
2017).

In this paper, we use EWI to invert for the medium parameter perturbations via a direct division (deconvolution) (Alkhalifah and Song, 2019). EWI is developed from WRI by formulating an optimization problem using an enhanced source function. By doing so, this new objective function becomes a linear optimization problem with respect to the wavefield and the modified source function. Using cheap inner iterations to update the wavefield and the modified source (no update the operator is needed), these two variables can be optimized. With a direct division, the medium parameter perturbations can be calculated directly. Since the proposed method can achieve good inversion results with fewer iterations, it is computationally more efficient than FWI and WRI. Thanks to the computational efficiency of EWI, we are able to sequentially invert for the three parameters. When one parameter gets updated, its update leakage will have less an effect on other parameter inversions, resulting in fewer tradeoff artifacts. Application on the anisotropic Marmousi model shows that the proposed approach can yield reasonable inverted medium parameter models in an efficient matter. A test on the 2D real dataset also shows the validity of the proposed method.

\section{THEORY}

Parameterization: $v_{n}, \eta, \delta$

To simulate wave propagation considering the anisotropic nature of the Earth, we use a VTI acoustic-wave equation with constant density expressed in the frequency domain (Zhou et al., 2006). The wave equation, parameterized using the normal moveout (NMO) velocity 
$v_{n}$ and anisotropic parameters $\delta$ and $\eta$, is stated as follows:

$$
\begin{aligned}
& -\frac{\omega^{2}}{v_{n}^{2}} p-\left(\frac{\partial^{2} p}{\partial x^{2}}+\frac{\partial^{2} q}{\partial x^{2}}\right)-\frac{1}{1+2 \delta} \frac{\partial^{2} p}{\partial z^{2}}=s \\
& -\frac{\omega^{2}}{v_{n}^{2}} q-2 \eta\left(\frac{\partial^{2} p}{\partial x^{2}}+\frac{\partial^{2} q}{\partial x^{2}}\right)=0 .
\end{aligned}
$$

For this VTI acoustic-wave equation, $p$ is the pressure wavefield, and $q$ is the perturbation wavefield. Here, $\omega$ denotes the angular frequency for wavefields in 2D media with coordinate variables $\mathrm{x}$ horizontally and z vertically. The frequency domain VTI acoustic-wave equations can be expressed in compact form as:

$$
\mathbf{L}(\mathbf{x}, \omega) u(\mathbf{x}, \omega)=f\left(\mathbf{x}_{\mathbf{s}}, \omega\right)
$$

where, $\mathbf{L}(\mathbf{x}, \omega)$ is the impedance matrix, which is also referred to as the modelling operator. $u(\mathbf{x}, \omega)=[p q]^{T}$ represents the wavefield vector, and $f(\mathbf{x}, \omega)=[s 0]^{T}$ is the source vector. $\mathbf{x}$ and $\mathbf{x}_{\mathbf{s}}$ denote the spatial dimensions and source locations. Usually, the sparse impedance matrix $\mathbf{L}(\mathbf{x}, \omega)$ is decomposed into a lower part $(\mathrm{L})$ and an upper part (U) using LU decomposition. As $\mathbf{L}(\mathbf{x}, \omega)$ is independent of the source function, the stored $\mathrm{L}$ and $\mathrm{U}$ matrices can be applied to different sources to improve for efficient wavefield calculation.

In the conventional FWI implementation, we tend to measure the misfit between predicted and observed data under the constraint of the wave equation:

$$
J(m)=\min \frac{1}{2} \sum_{i}\left\|d_{i}-C u_{i}\right\|_{2}^{2} \quad \text { s.t. } \quad \mathbf{L}(m) u_{i}=f_{i},
$$

where, $i$ is the source index, $d_{i}$ is recorded data, and $m$ is the medium parameters. Based on the adjoint-state method (R.E.Plessix, 2006), the velocity gradient is given by the crosscorrelation between the background and adjoint wavefields. The adjoint wavefield is calculated by back propagating the data residual at the sensors locations. The biggest challenge in conventional FWI is the large computational cost and memory requirements in storing the background wavefields. The wavefield $u$ is calculated using the wave equation, 
which depends on the medium parameters. This dependency causes the non-linearity of the objective function. In multi-parameter waveform inversion, we have another level of complexity. As the data residuals may come from different medium parameter perturbations, gradient-based optimization methods will suffer from the tradeoff between different parameters (Gholami et al., 2013). In order to solve these problems, we propose to use EWI to achieve multi-parameter inversion in acoustic VTI media. The objective function of EWI is given by:

$$
E\left(u_{i}, f_{e i}\right)=\min \frac{1}{2} \sum_{i}\left\|d_{i}-C u_{i}\right\|_{2}^{2}+\frac{\alpha^{2}}{2}\left\|\mathbf{L}_{\mathbf{0}} u_{i}-f_{e i}\right\|_{2}^{2},
$$

where $\alpha^{2}$ is a weighting parameter balancing the data fitting term and the wave equation term. At the beginning of the inversion process, we start with a background VTI modeling operator $\mathbf{L}_{\mathbf{0}}$, which corresponds to the background medium parameters: $v_{h 0}, \epsilon_{0}, \eta_{0}$. In the VTI acoustic-wave equation, wavefield vector $u=[p q]^{T}$ consists of the pressure wavefield $p$ and perturbation wavefield $q$. In equation 4 , the modified source function $f_{e}=\left[f_{e p} f_{e q}\right]^{T}$ starts with the original source function $f=\left[\begin{array}{ll}s & 0\end{array}\right]^{T}$ in the inversion process. Here, $f_{e}$ is a function of the space and the frequency, $d$ represents the observed data, and the mapping operator $C$ just extracts values from the pressure wavefield $q$ at the receiver positions and ignores the perturbation wavefield $p$ which is an auxiliary function. In order to calculate the wavefield, which needs to simultaneously satisfy the data fitting objective and the wave equation, we minimize $E$ by solving $\nabla_{u} E=0$. Thus, the wavefield satisfies the following linear equation:

$$
\left(\begin{array}{c}
\alpha \mathbf{L}_{0} \\
C
\end{array}\right) u_{i}=\left(\begin{array}{c}
\alpha f_{e i} \\
d_{i}
\end{array}\right)
$$

We solve this linear equation using a least-squares approach to obtain the estimated wavefield $u=\left[\begin{array}{ll}p & q\end{array}\right]^{T}$ (Alkhalifah and Song, 2019). This extended augmented wave equation reconstructs the initial pressure wavefield $p$ and perturbation wavefield $q$ in the model 
space using the background medium parameters. This background-medium wavefield will include only single scattered energy from the missing perturbations. However, such energy often dominates the wavefield, compared to multi scattering. The modified source function satisfying the wave equation can be calculated using $\mathbf{L}_{\mathbf{0}}$ as:

$$
f_{e}=\mathbf{L}_{\mathbf{0}} u \text {. }
$$

We set $m_{n}=\frac{1}{v_{n}^{2}}, \zeta=\frac{1}{1+2 \delta}$, and define the initial (background) medium parameters with $m_{0}, \zeta_{0}$ and $\eta_{0}$. Using the background medium parameters, equation 1 is expressed as:

$$
\begin{aligned}
& -\omega^{2} m_{n 0} p-\left(\frac{\partial^{2} p}{\partial x^{2}}+\frac{\partial^{2} q}{\partial x^{2}}\right)-\zeta_{0} \frac{\partial^{2} p}{\partial z^{2}}=f_{e p}, \\
& -\omega^{2} m_{n 0} q-2 \eta_{0}\left(\frac{\partial^{2} p}{\partial x^{2}}+\frac{\partial^{2} q}{\partial x^{2}}\right)=f_{e q} .
\end{aligned}
$$

The modified source function $f_{e}=\left[\begin{array}{ll}f_{e p} & f_{e q}\end{array}\right]^{T}$ satisfies the wave equation using the background operator $\mathbf{L}_{\mathbf{0}}$. Using the new $f_{e}$, we can solve for the wavefield again to include multiscattered energy. We define the medium perturbations by $\delta m_{n}, \delta \zeta$, and $\delta \eta$, so the true models are given by:

$$
m_{n}=m_{n 0}+\delta m_{n} ; \zeta=\zeta_{0}+\delta \zeta ; \eta=\eta_{0}+\delta \eta
$$

Plugging equations 8 into equation 1 and subtracting equation 7 , we get the relations between medium parameter perturbations and the modified source as follows:

$$
\begin{aligned}
& s+\omega^{2} \delta m_{n} p+\delta \zeta \frac{\partial^{2} p}{\partial z^{2}}=f_{e p}, \\
& \omega^{2} \delta m_{n} q+2 \delta \eta\left(\frac{\partial^{2} p}{\partial x^{2}}+\frac{\partial^{2} q}{\partial x^{2}}\right)=f_{e q} .
\end{aligned}
$$

In the implementation of this multi-parameter inversion, we invert for each medium parameter perturbation in the order of $v_{n}, \eta, \delta$, sequentially. We always update the velocity first, because velocity is the dominant parameter controlling the wave propagation in this 
parameterization. By doing so, the velocity update leakage to the anisotropic parameters is reduced. Equation 9 shows that $\delta m_{n}$ has dependences on both pressure wavefield $p$ and perturbation wavefield $q$. As perturbation wavefield $q$ is mainly affected by the anisotropic parameters, we do not include $q$ in the squared slowness perturbation calculation. In the sequential update scheme, when we update one target parameter, we assume the perturbations of other parameters are zero. As a result, the medium parameter perturbations of $\delta m, \delta \zeta$ and $\delta \eta$ can be calculated using a direct division as:

$$
\begin{aligned}
& \delta m_{n}=\Re\left[\frac{p^{*}\left(s-f e_{p}\right)}{\omega^{2} p^{*} p+\lambda}\right], \\
& \delta \zeta=\Re\left[\frac{w^{*}\left(s-f e_{p}\right)}{\omega^{2} w^{*} w+\lambda}\right], \\
& \delta \eta=\Re\left[\frac{r^{*} f e_{q}}{\omega^{2} r^{*} r+\lambda}\right],
\end{aligned}
$$

where, $\lambda$ is a small value to avoid division by zero, $w=\frac{\partial^{2} p}{\partial z^{2}}$, and $r=\frac{\partial^{2} p}{\partial x^{2}}+\frac{\partial^{2} q}{\partial x^{2}}$. After obtaining the parameter perturbations $\delta m, \delta \zeta$ and $\delta \eta$, we update the background model using equation 8 . There is also another way to invert for the three parameters simultaneously by formulating equation 9 into matrix and vector multiplication for each image point. This method is used in isotropic elastic media to invert for the P- and S-wave velocities (Song et al., 2019a). The reason they use this method is because P-and S-wave velocities equally contribute to the wave propagation in the elastic media. However, this method is computationally expensive for a large model. In acoustic VTI media, velocity is the dominant parameter, and the anisotropic parameters have relatively milder influences on the wave propagation. Thus, we use a direct division approach to calculate the three parameters, sequentially.

In our implementation, we conduct the inversion process in the frequency domain. We use inner iterations to update $u=\left[\begin{array}{ll}p & q\end{array}\right]^{T}$ and $f_{e}=\left[\begin{array}{ll}f_{e p} & f_{e q}\end{array}\right]^{T}$, efficiently. As a result, $f_{e}$ 
contains the perturbations in medium parameters. We plug the calculated $f_{e}$ back into equation 5 , so the parameter perturbations in $f_{e}$ act as secondary sources in reconstructing the wavefield. As a result, multi-scattering components are added to the wavefield within every inner iteration (Alkhalifah and Song, 2019). As parameter perturbations calculation will benefit from multi-scattering components, the convergence rate in each parameter inversion accelerates (Alkhalifah and Wu, 2016; Song et al., 2019b). The detailed algorithm is explained in the Algorithm 1.

\section{Parameterization: $v_{h}, \eta, \epsilon$}

As the horizontal velocity $v_{h}$, the NMO velocity $v_{n}$, and Thomsen anisotropy parameters $\delta, \eta, \epsilon$ have the following relations:

$$
v_{h}=v_{n} \sqrt{1+2 \eta}, \quad 1+2 \delta=\frac{1+2 \epsilon}{1+2 \eta} .
$$

We re-parameterize the VTI acoustic-wave equation 1 using the horizontal velocity $v_{h}$ and anisotropic parameters $\epsilon$ and $\eta$. As a result, the VTI acoustic-wave equation is given by:

$$
\begin{aligned}
& -\frac{\omega^{2}}{v_{h}^{2}} p-\frac{1}{1+2 \eta}\left(\frac{\partial^{2} p}{\partial x^{2}}+\frac{\partial^{2} q}{\partial x^{2}}\right)-\frac{1}{1+2 \epsilon} \frac{\partial^{2} p}{\partial z^{2}}=s, \\
& -\frac{\omega^{2}}{v_{h}^{2}} q-\frac{2 \eta}{1+2 \eta}\left(\frac{\partial^{2} p}{\partial x^{2}}+\frac{\partial^{2} q}{\partial x^{2}}\right)=0 .
\end{aligned}
$$

We set $m=\frac{1}{v_{h}^{2}}, \xi=\frac{1}{1+2 \eta}, \mu=\frac{1}{1+2 \epsilon}$, and perturb each medium parameter by $\delta m, \delta \xi$ and $\delta \mu$. Using the background medium parameters, equation 14 is expressed as:

$$
\begin{aligned}
& -\omega^{2} m_{h 0} p-\xi_{0}\left(\frac{\partial^{2} p}{\partial x^{2}}+\frac{\partial^{2} q}{\partial x^{2}}\right)-\mu_{0} \frac{\partial^{2} p}{\partial z^{2}}=f_{e p}, \\
& -\omega^{2} m_{h 0} q+\xi_{0}\left(\frac{\partial^{2} p}{\partial x^{2}}+\frac{\partial^{2} q}{\partial x^{2}}\right)-\left(\frac{\partial^{2} p}{\partial x^{2}}+\frac{\partial^{2} q}{\partial x^{2}}\right)=f_{e q} .
\end{aligned}
$$


Subtracting equation 15 from equation 14, we get the relations between medium parameter perturbations and the modified source shown as:

$$
\begin{aligned}
& s+\omega^{2} \delta m_{h} p+\delta \xi\left(\frac{\partial^{2} p}{\partial x^{2}}+\frac{\partial^{2} q}{\partial x^{2}}\right)+\delta \mu \frac{\partial^{2} p}{\partial z^{2}}=f_{e p} \\
& \omega^{2} \delta m_{n} q-\delta \xi\left(\frac{\partial^{2} p}{\partial x^{2}}+\frac{\partial^{2} q}{\partial x^{2}}\right)=f_{e q} .
\end{aligned}
$$

Using the similar idea in the previous parameterization, the medium parameter perturbations of $\delta m_{h}, \delta \xi$ and $\delta \mu$ are calculated using:

$$
\delta m_{h}=\Re\left[\frac{p^{*}\left(s-f_{e p}\right)}{\omega^{2} p^{*} p+\lambda}\right], \delta \mu=\Re\left[\frac{w^{*}\left(s-f_{e p}\right)}{w^{*} w+\lambda}\right], \delta \xi=\Re\left[\frac{r^{*} f_{e q}}{r^{*} r+\lambda}+\frac{r^{*}\left(s-f_{e p}\right)}{r^{*} r+\lambda}\right],
$$

where, $w=\frac{\partial^{2} p}{\partial z^{2}}$ and $r=\frac{\partial^{2} p}{\partial x^{2}}+\frac{\partial^{2} q}{\partial x^{2}}$. After obtaining the anisotropic parameters perturbation $\delta m_{h}, \delta \mu$ and $\delta \xi$, the updated anisotropic parameters are evaluated using:

$$
\begin{aligned}
& m_{h 0}=m_{h 0}+\delta m_{h}, v_{h 0}=\sqrt{\frac{1}{m_{h 0}}} \\
& \xi_{0}=\xi_{0}+\delta \xi, \eta_{0}=\left(\frac{1}{\xi_{0}}-1\right) / 2, \\
& \mu_{0}=\mu_{0}+\delta \mu, \epsilon_{0}=\left(\frac{1}{\mu_{0}}-1\right) / 2 .
\end{aligned}
$$

\section{NUMERICAL EXAMPLES}

\section{Gaussian anomaly model}

We include in the horizontal velocity of a VTI model a Gaussian anomaly on the right-hand side of a homogeneous background, and in the $\eta$ model another Gaussian anomaly on the left-hand side of a homogeneous background, as shown in Figures 1a and 1b. We ignore the influence of the anisotropic parameter $\epsilon$, and set it to zero. The background velocity and $\eta$ are $2 \mathrm{~km} / \mathrm{s}$ and zero, respectively. We place 20 sources on the surface and receivers on all the boundaries to have a full aperture acquisition system. We use a single frequency of 5 $\mathrm{Hz}$ to conduct a multi-parameter EWI inversion process for the velocity and $\eta$, sequentially. 
The source function we use to generate the synthetic data is a delta function. The $\alpha^{2}$ we use in all examples in this paper is $10 e^{8}$, as chosen based on trial and error tests. After 20 iterations of updates to horizontal velocity first, and $\eta$ second, the inversion results are shown in Figures 1c and 1d. We use two inner iterations in every model update iteration. We observe that the horizontal velocity is very well recovered even with one single frequency, and $\eta$ is partially resolved in the correct position. To understand the inversion results, we show the radiation patterns for this parametrization $\left(v_{h}, \eta, \epsilon\right)$ in Figure 2. We observe that different parameters have different sensitivities to the data, unlike the horizontal velocity $v_{h}, \eta$ is only sensitive to mid-range scattering angles using this parameterization. The results show that EWI can obtain reasonable inversion results of their unique positions with minimal tradeoff. To demonstrate the superiority of the proposed approach, we implement a conventional multi-parameter FWI for acoustic VTI media with the same inversion setup (Djebbi and Alkhalifah, 2018), and the inversion results of horizontal velocity and $\eta$ are shown in Figures 3a and 3b, respectively. It is obvious that the horizontal velocity inverted by conventional FWI is not as accurate as the inverted horizontal velocity by EWI. In the inverted $\eta$ model by conventional FWI, there are strong artifacts around the location of the horizontal velocity anomaly. By comparison, the inverted $\eta$ model by EWI is generally clean with fewer artifacts caused by the tradeoff issue.

\section{D anisotropic Marmousi model}

We further apply the multi-parameter inversion using EWI on the anisotropic Marmousi model. First, we use the parameterization $v_{n}, \eta$ and $\delta$. The true NMO velocity model is shown in Figure 4a, and the true anisotropic parameter model is shown in Figure 4b. We use the same model for $\eta$ and $\delta$. We use 36 sources on the surface, which are denoted by 
* symbol in Figure 4a. All the surface grid points act as receivers. We use an $8 \mathrm{~Hz}$ Ricker wavelet as our source function. The Ricker wavelet in the time domain and its frequency magnitude spectrum are shown in in Figures 5a and 5b, respectively. We transform the time-domain wavelet into frequency domain to generate the synthetic data by solving the Helmholtz wave equation. The initial velocity model is linearly increasing with depth model as shown in Figure 6a, and the initial $\eta$ and $\delta$ model is homogeneously zero as shown in Figure 6b. In the implementation, we first invert for the velocity, then we invert for $\eta$, and finally for $\delta$. For each parameter inversion, we use two inner iterations. Within each frequency, the iteration number for the NMO velocity $v_{n}$, and anisotropic parameter $\eta$ and $\delta$ are 4,3 , and 3 , respectively. The selected frequency band extends from $2 \mathrm{~Hz}$ to $10 \mathrm{~Hz}$ with the sampling interval of $0.5 \mathrm{~Hz}$. We start the inversion from low to high frequency. We generate the synthetic data in the frequency domain using the true models in Figure 4. The inversion results for the NMO velocity $v_{n}, \eta$ and $\delta$ are shown in Figures 7a-7c, respectively.

From the inversion results, we see that the NMO velocity $v_{n}$ is very well resolved. However, when we compare the velocities using a vertical profile at $3.7 \mathrm{~km}$ (shown in Figure 8), the inverted NMO velocity is slightly shifted in depth from the true one. The lowwavenumber components of the $\eta$ model in the shallow part are recovered, as $\eta$ is more sensitive to the large scattering angles. As for the $\delta$ model, the high-wavenumber components corresponding to reflectors are inverted. The radiation patterns of $v_{n}, \eta, \delta$ are shown in Figure 9. It provides theoretical insights into the potential tradeoff between the resolved parameters (Alkhalifah and Plessix, 2014). The NMO velocity has an angle independent radiation pattern. On the other hand, $\eta$ is mainly provided by large scattering angles or large offsets. Finally, $\delta$ is more sensitive to small scattering angles which correspond to reflection waves. 
We next test EWI with the parametrization $\left(v_{h}, \eta, \epsilon\right)$ on the same models. The true horizontal velocity $v_{h}$, and anisotropic parameter model $\eta$ and $\epsilon$ are shown in Figures 4 a, and $4 \mathrm{~b}$, respectively. The source and receiver setup is the same as the previous example. We regenerate the synthetic data using the same source function used with the previous parameterization. Using the same initial models, we again first invert for the horizontal velocity, then we invert for $\eta$ and $\epsilon$. With the same frequency band and inversion strategy, the inversion results of horizontal velocity $v_{h}, \eta$ and $\epsilon$ are shown in Figures 10a-10c, respectively. We also perform a conventional multi-parameter FWI for acoustic VTI media using the same inversion setup and parameterization (Djebbi and Alkhalifah, 2018), and the inversion results of the horizontal velocity, $\eta$ and $\epsilon$ are shown in Figures 11a-11c, respectively. The vertical profile at $3.7 \mathrm{~km}$ is shown in Figure 12, and it indicates that the inverted horizontal velocity using EWI fits the true velocity very well. By comparison, the inverted horizontal velocity using FWI has a poor match with the true horizontal velocity. Using this parametrization, the horizontal velocity $v_{h}$ still has an isotropic radiation pattern, and as a result, it can be recovered very well at most scales. On the other hand, $\epsilon$ is more sensitive to the small scattering angles and can only be resolved at the finer scale. So only the high wave-number components of $\epsilon$ are reconstructed, and this is more obvious from the inverted $\epsilon$ model using EWI. By comparison, $\eta$ has much lower sensitivity than the other two parameters, so $\eta$ can only be recovered partially and mostly up shallow as Alkhalifah (2016) predicted. Between these two parameterizations, the parameterization: $v_{h}, \eta, \epsilon$ is the optimal parameterization for waveform-based inversion (Alkhalifah and Guitton, 2016). In this parameterization, $\eta$ plays a minor role in FWI, while $\epsilon$ absorbs some of the amplitude mismatch, and thus, captures some of the reflectivity information. 


\section{D Real data}

This 2-D marine dataset is from North Western Australia Continental Shelf, acquired by CGG using a Broadseis acquisition system with a variable depth streamer (Soubaras and Dowle, 2010). Frequencies below $2.5 \mathrm{~Hz}$ have been filtered out due to the poor signal-tonoise ratio (SNR). One example shot gather is shown in Figure 13. The original dataset contains 1824 shot gathers at an interval of approximately $0.01875 \mathrm{~km}$, we choose only 100 shot gathers corresponding to the $2 \mathrm{D}$ survey area of interest. The target model area we choose is $12.5 \mathrm{~km}$ long and $3.75 \mathrm{~km}$ in depth. We perform EWI using the parameterization $v_{h}, \eta, \epsilon$. The initial horizontal velocity model and the estimated wavelet are borrowed from (Kalita and Alkhalifah, 2017, 2018), as shown in Figures 14 and 15. The initial anisotropic parameters $\eta$ and $\epsilon$ are zero. We resample the data and use 324 receivers, with an interval of $0.025 \mathrm{~km}$.

Firstly, we perform an isotropic EWI in two stages by a hierarchical approach. The frequency spectrum of the two selected frequency bands is shown in Figure 16. In stage 1, we invert for the selected frequency band from $3 \mathrm{~Hz}$ to $6 \mathrm{~Hz}$ with a dense sampling of $0.15 \mathrm{~Hz}$. In stage 2, the selected frequency band is from $9 \mathrm{~Hz}$ to $12 \mathrm{~Hz}$. We use two inner iterations within each frequency and two outer iterations over the frequencies of each stage. The inversion result is shown in Figure 17. It is obvious that more detailed highresolution information is recovered in the velocity model, especially in low velocity layers between $2.0 \mathrm{~km}$ to $2.5 \mathrm{~km}$ depth. Next, we perform an anisotropic EWI using the same inversion setup and strategy. We use the isotropic inverted velocity model as the initial velocity, and set initial anisotropic parameters to zero. We test anisotropic EWI with the parametrization using $\left(v_{h}, \eta, \epsilon\right)$ with the same setup. The inverted horizontal velocity, $\eta$, 
and $\epsilon$ are shown in Figures 18a-18c, respectively. We see that low-wavenumber $\eta$ is recovered in the shallow part, while high-wavenumber $\epsilon$ is recovered throughout. The vertical velocity profile corresponding to check-shot measurement at $10.5 \mathrm{~km}$ is shown in Figure 19, and the smooth velocity between $1 \mathrm{~km}$ and $2 \mathrm{~km}$ is obtained through interpolation, which does not reflect measurements. It shows that the inverted horizontal velocity model from the acoustic VTI EWI fits the well-log velocity reasonably well in the high velocity layers at the depths of $2.2 \mathrm{~km}$ and $3.2 \mathrm{~km}$, and also the low velocity layer at the depth of $3.4 \mathrm{~km}$. According to the radiation patterns shown in Figure 2, the horizontal velocity has angle independent radiation pattern. $\epsilon$ is more sensitive to small scattering angles, which correspond to reflection waves. While $\eta$ has a much lower sensitivity when the horizontal velocity is used, and it can only be recovered partially in the shallow part. We perform reverse time migration (RTM) using the initial and inverted models, and the images are shown in Figure 20. Figure 20b shows that multiple events (as the red arrows point out) are highlighted using the inverted models. The events at depths between $2.5 \mathrm{~km}$ and $3 \mathrm{~km}$ are better resolved with higher resolution in the RTM image corresponding to the inverted models. Finally, we compare the 50th observed shot gather with the predicted data generated from the initial models and wavelet, which is shown in Figure 21. It is obvious that the predicted data using the initial models lacks most of the reflection events apparent in observed data, and there is a clear mismatch in the diving wave. We use the inverted models to invert for the source wavelet in the time domain, and the result is shown in Figure 22. Using the inverted model and wavelet, the predicted data recovers the reflection waves seen in the observed data, and the diving waves match the observed data reasonably well, as shown in Figure 23. 


\section{DISCUSSIONS}

We apply a multi-parameter waveform inversion using efficient wavefield inversion (EWI) in acoustic VTI media. The key point of EWI is the inner iteration between the wavefield $u$ and the modified source $f_{e}$. The wavefield would initially contain only single scattering components from the missing perturbations, while secondary sources (medium parameter perturbations) reside in $f_{e}$. We can apply additional regularizations on $f_{e}$ to enhance certain features and mitigate the non-uniqueness in the solutions (Liu et al., 2015; Li et al., 2017; Lu et al., 2018; Kalita et al., 2019). Inner iterations add these additional scattering components to the wavefield. Specifically, another iteration of the linear inversion for $u$, with the newly obtained $f_{e}$ will add some energy of the second-order scattering to the wavefield. Theoretically, the more inner iterations, the more scattering information will be added to the wavefield. In practice, scattering energy higher than the second order is often weak. So two inner iterations is sufficient to calculate accurate model perturbations. The detailed discussions of inner iterations is introduced in Alkhalifah and Song (2019).

In inverting for the wavefield $u$, the weighting factor $\alpha^{2}$ plays an important role in avoiding cycle-skipping. The higher the $\alpha^{2}$ we use, the more the emphasis is put into fitting the wave equation. In this case, the data fitting is reduced, and we face the potential for cycle skipping when the initial velocity is poor. On the other hand, if we use small $\alpha^{2}$, the accuracy of the wave equation is relaxed and data fitting is enhanced. As a result, the cycle-skipping problem can be somewhat mitigated. From our experience, it would be a good idea to start with a relatively small $\alpha^{2}$. Detailed analysis of the influence of $\alpha^{2}$ on EWI is discussed in Alkhalifah and Song (2019).

A simultaneous update scheme for all parameters may suffer from tradeoff issues due 
to velocity update leakage (Li et al., 2018). Oh et al. (2018) used a hierarchical inversion for ocean bottom cable field data. They start their inversion with an acoustic FWI, and gradually include more anisotropic parameters in late stages of the inversion. In our implementation, we use a sequential inversion scheme to invert for the velocity and the anisotropic parameters. The extra cost is affordable, because EWI does not require the calculation of the adjoint wavefield step, which is inevitable in FWI. In addition, the velocity perturbation includes the multiscattering information in EWI, so it requires fewer number of iterations to achieve convergence (Alkhalifah and Song, 2019). Thus, EWI is more efficient than FWI for acoustic VTI media.

\section{CONCLUSIONS}

We apply an efficient wavefield inversion (EWI) to implement multi-parameter inversion in acoustic VTI media using various parameterizations. We invert for the three medium parameters in a sequential matter. A test on a model with distinct anomalies for each parameter shows that acoustic VTI EWI can invert for the velocity and the anisotropic parameters with limited tradeoff artifacts. Applications on synthetic data generated from the anisotropic Marmousi model show that EWI can recover the medium parameters that are predicted by the radiation patterns for the corresponding parametrization. The application to field data helped us recover a reasonably high resolution velocity model that managed to predict the data accurately.

\section{ACKNOWLEDGMENTS}

We thank KAUST for sponsoring this research. We thank our SWAG colleagues for their helpful suggestions, especially Mahesh Kalita for providing the initial velocity model. We 
also thank CGG for providing the field data set and Geoscience Australia for providing the well-log information. We also thank the editor Jeffrey Shragge, the associate editor Aria Abubakar, and two anonymous reviewers for their important suggestions and comments. This work utilized the resources of the Supercomputing Laboratory at King Abdullah University of Science and Technology (KAUST) in Thuwal, Saudi Arabia, and we are grateful for that. 


\section{REFERENCES}

Abubakar, A., W. Hu, T. Habashy, and M. P. B. van, 2009, Application of the finitedifference contrast-source inversion algorithm to seismic full-waveform data: Geophysics, 74, no.6, WCC47-WCC58.

Alkhalifah, T., 2016, Research note: Insights into the data dependency on anisotropy: An inversion prospective: Geophysical Prospecting, 64, 505-513.

— 2019, Linear wavefield optimization using a modified source: Communications in computational physics, doi: 10.4208/cicp.OA-2018-0087.

Alkhalifah, T., and A. Guitton, 2016, An optimal parameterization for full waveform inversion in anisotropic media: 78th EAGE Conference and Exhibition 2016, We SRS2 05.

Alkhalifah, T., and R.-É. Plessix, 2014, A recipe for practical full-waveform inversion in anisotropic media: An analytical parameter resolution study: Geophysics, 79, no.3, R91R101.

Alkhalifah, T., and C. Song, 2019, An efficient wavefield inversion: Using a modified source function in the wave equation: Geophysics, 84, no.6, R921-R934.

Alkhalifah, T., and Z. Wu, 2014, Fwi and mva the natural way: 74th Annual International Conference and Exhibition, EAGE, Extend Abstracts, WeE10612.

—_, 2016, Multiscattering inversion for low-model wavenumbers: Geophysics, 81, no.6, R417-R428.

Djebbi, R., and T. Alkhalifah, 2018, Frequency domain multiparameter acoustic inversion for transversely isotropic media with a vertical axis of symmetry: Geophysics, 84, no.1, C1-C14.

Djebbi, R., R.-É. Plessix, and T. Alkhalifah, 2017, Analysis of the traveltime sensitivity 
kernels for an acoustic transversely isotropic medium with a vertical axis of symmetry: Geophysical Prospecting, 65, 22-34.

Gholami, Y., R. Brossier, S. Operto, A. Ribodetti, and J. Virieux, 2013, Which parameterization is suitable for acoustic vertical transverse isotropic full waveform inversion? part 1: Sensitivity and trade-off analysis: Geophysics, 78, no.2, R81-R105.

Guitton, A., and T. Alkhalifah, 2017, A parameterization study for elastic vti full-waveform inversion of hydrophone components: Synthetic and north sea field data examplesparameterization: Geophysics, 82, R299-R308.

Kalita, M., and T. Alkhalifah, 2017, Efficient full waveform inversion using the excitation representation of the source wavefield: Geophysical Journal International, 210, 15811594.

, 2018, Multiscale full-waveform inversion using flux corrected transport: SEG Technical Program Expanded Abstracts, 1153-1157.

Kalita, M., V. Kazei, Y. Choi, and T. Alkhalifah, 2019, Regularized full-waveform inversion with automated salt flooding: Geophysics, 84, no.4, R569-R582.

Leeuwen, T. V., and F. Herrmann, 2013, Mitigating local minima in full-waveform inversion by expanding the search space: Geophysical Journal International, 195, 661-667.

—, 2015 , A penalty method for pde-constrained optimization in inverse problems: Inverse Problems, 32, 015007.

Li, C., J.-P. Huang, Z.-C. Li, and R.-R. Wang, 2017, Regularized least-squares migration of simultaneous-source seismic data with adaptive singular spectrum analysis: Petroleum science, 14, 61-74.

Li, Y., T. Alkhalifah, and H. Gu, 2018, Using well information to verify and improve waveform inversion results in transversely isotropic media: Presented at the 80th EAGE 
Conference and Exhibition 2018.

Liu, C., C. Song, Q. Lu, Y. Liu, X. Feng, and Y. Gao, 2015, Impedance inversion based on 11 norm regularization: Journal of Applied Geophysics, 120, 7-13.

Lu, Q., C. Song, and C. Liu, 2018, A new prestack three-parameter amplitude variation with offset inversion method: Journal of Geophysics and Engineering, 15, 1300-1309.

Oh, J.-W., M. Kalita, and T. Alkhalifah, 2018, 3D elastic full-waveform inversion using p-wave excitation amplitude: Application to ocean bottom cable field data: Geophysics, 83, no.2, R129-R140.

R.E.Plessix, 2006, A review of the adjoint-state method for computing the gradient of a functional with geophysical applications: Geophysical Journal International, 167, 495503.

Song, C., T. Alkhalifah, G. Wang, and Q. Yang, 2019a, An efficient wavefield inversion for isotropic elastic media, in SEG Technical Program Expanded Abstracts 2019: Society of Exploration Geophysicists, 1575-1579.

Song, C., Z. Wu, and T. Alkhalifah, 2019b, Passive seismic event estimation using multiscattering waveform inversion: Geophysics, 84, no.3, KS59-KS69.

Soubaras, R., and R. Dowle, 2010, Variable-depth streamer-a broadband marine solution: first break, 28 .

Tarantola, A., 1984, Inversion of seismic reflection data in the acoustic approximation: Geophysics, 49, 1259-1266.

Virieux, J., and S. Operto, 2009, An overview of full-waveform inversion in exploration geophysics: Geophysics, 74, no.6, WCC1-WCC26.

Wang, C., D. Yingst, P. Farmer, I. Jones, G. Martin, and J. Leveille, 2016, Full-waveform inversion with the reconstructed wavefield method: SEG Technical Program Expanded 
Abstracts 2016, 1237-1241.

_ 2017, Reconstructed full-waveform inversion with the extended source: SEG Technical Program Expanded Abstracts 2017, 1449-1453.

Zhou, H., G. Zhang, and R. Bloor, 2006, An anisotropic acoustic wave equation for vti media: 68th EAGE Conference and Exhibition incorporating SPE EUROPEC 2006, H033. 


\section{LIST OF FIGURES}

1 The true horizontal velocity model (a), anisotropic parameter $\eta$ model (b), the inverted horizontal velocity model (c), and anisotropic parameter $\eta$ model (d).

2 Radiation patterns for the parametrization $\left(v_{h}, \eta, \epsilon\right)$.

3 The inverted horizontal velocity model (a), and anisotropic parameter $\eta$ model (b) using conventional FWI.

4 The true Marmousi velocity model with source geometry (a) and the true anisotropic parameter model (b).

5 The Ricker wavelet (a), and the frequency spectrum corresponding to it (b).

6 Initial NMO (horizontal) velocity $v_{n}(\mathrm{a})$, and anisotropic parameter model (b).

$7 \quad$ Inverted NMO velocity $v_{n}(\mathrm{a}), \eta(\mathrm{b})$ and $\delta(\mathrm{c})$.

8 A vertical NMO velocity profile at $3.7 \mathrm{~km}$.

9 Radiation patterns for the parametrization $\left(v_{n}, \eta, \delta\right)$.

10 Inverted horizontal velocity $v_{h}(\mathrm{a}), \eta(\mathrm{b})$ and $\epsilon(\mathrm{c})$ using EWI.

11 Inverted horizontal velocity $v_{h}(\mathrm{a}), \eta(\mathrm{b})$ and $\epsilon$ (c) using FWI.

12 A vertical horizontal velocity profile at $3.7 \mathrm{~km}$.

13 A shot gather from the real dataset.

14 The initial velocity model.

15 The estimated wavelet using the initial model.

16 The frequency spectrum of the two selected frequency bands.

17 The inverted velocity using isotropic EWI.

18 The horizontal velocity $v_{h}(\mathrm{a}), \eta(\mathrm{b})$, and $\epsilon(\mathrm{c})$.

19 The horizontal velocity profile at $10.5 \mathrm{~km}$ compared to the well-log vertical velocity.

20 The RTM image for the initial (a) and inverted (b) models (the red arrows points 
to areas of clear improvements).

21 A shot gather displaying interleaved predicted (Pre) and observed (Obs) data using the initial model.

22 The estimated wavelet using inverted model.

23 A shot gather displaying interleaved predicted (Pre) and observed (Obs) data using the inverted model. 

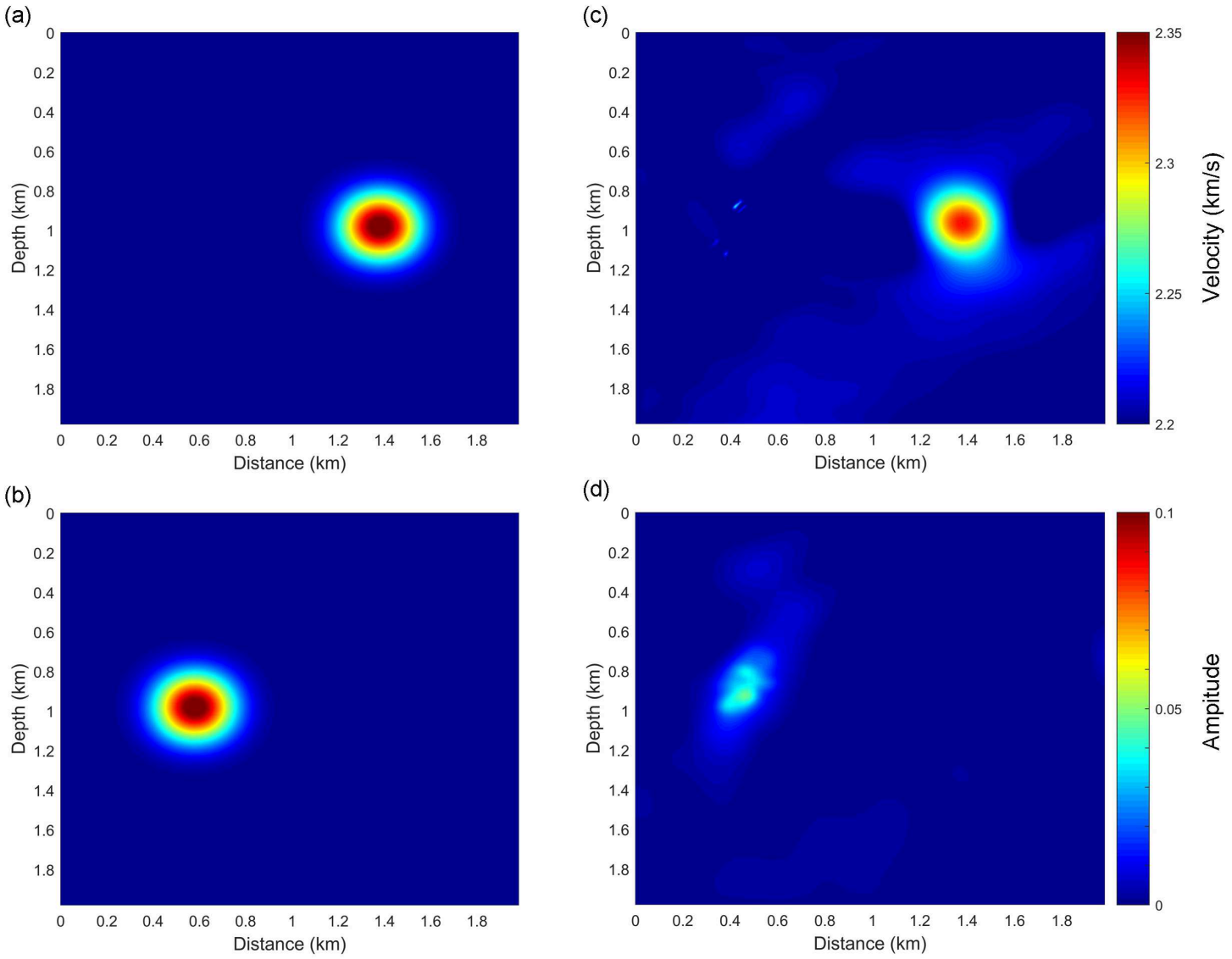

Figure 1: The true horizontal velocity model (a), anisotropic parameter $\eta$ model (b), the inverted horizontal velocity model (c), and anisotropic parameter $\eta$ model (d). 


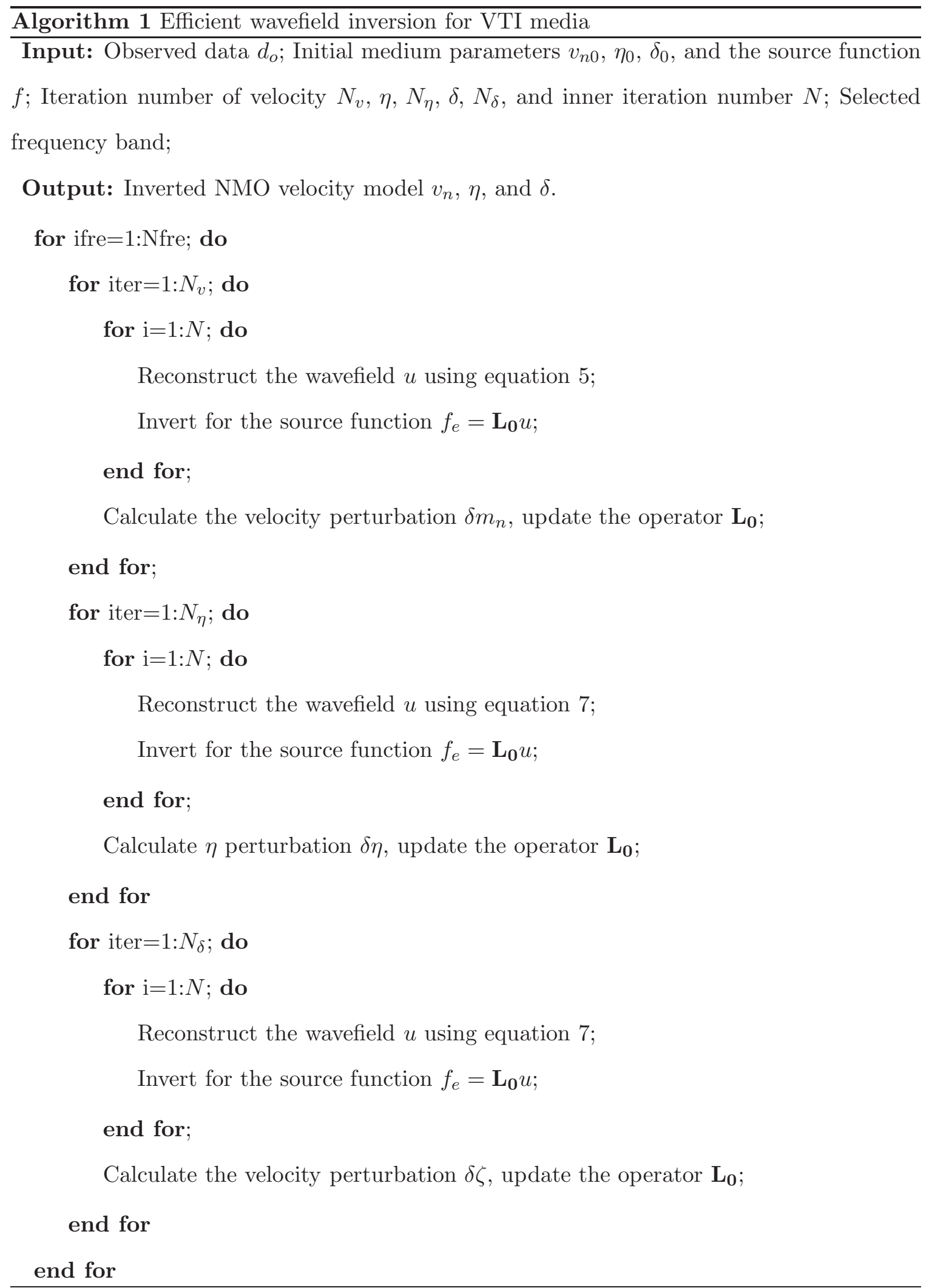




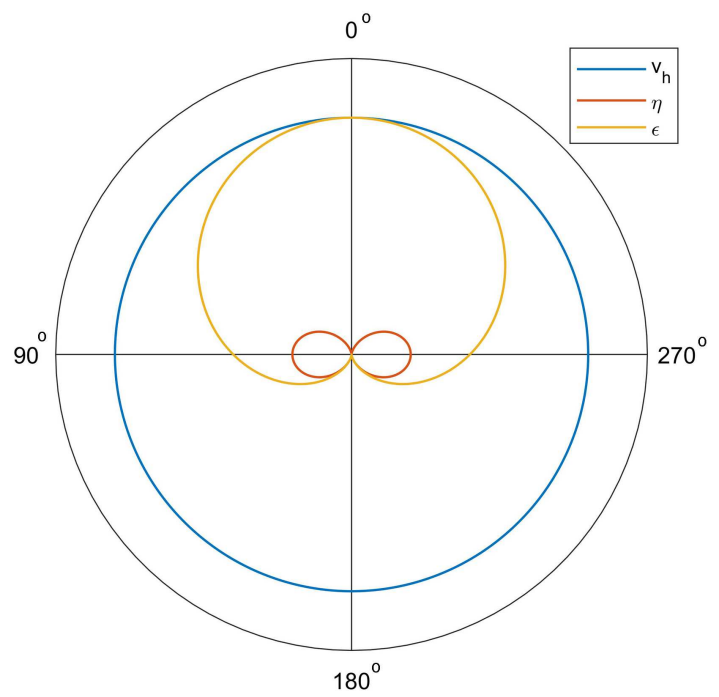

Figure 2: Radiation patterns for the parametrization $\left(v_{h}, \eta, \epsilon\right)$. - 

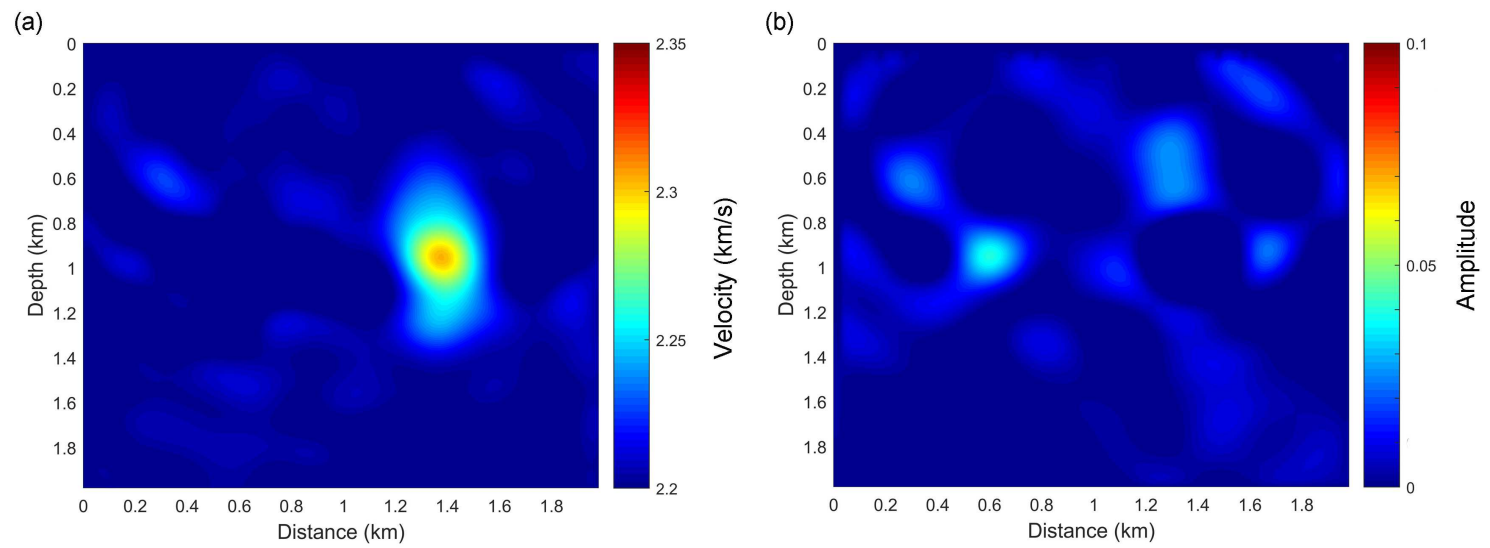

Figure 3: The inverted horizontal velocity model (a), and anisotropic parameter $\eta$ model (b) using conventional FWI. 

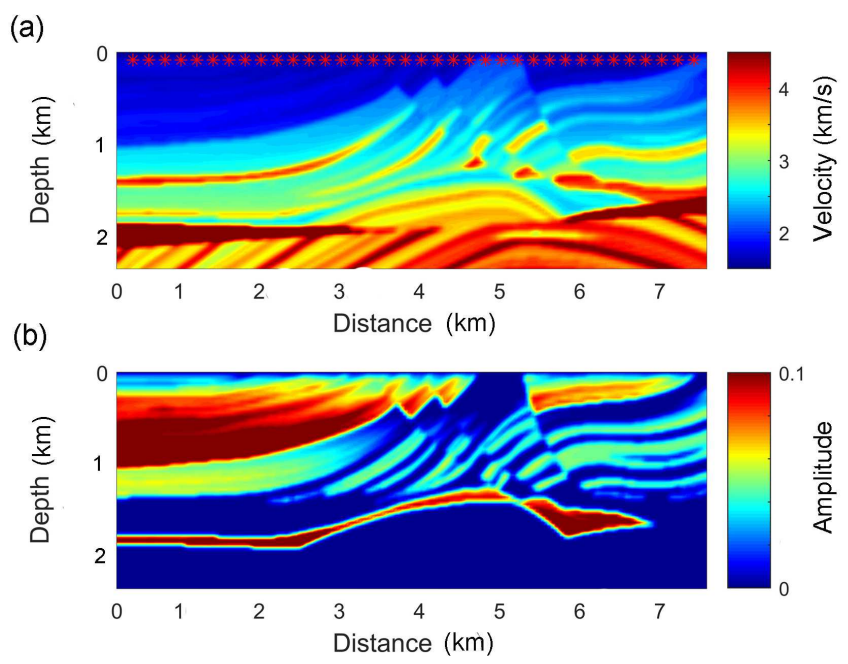

Figure 4: The true Marmousi velocity model with source geometry (a) and the true anisotropic parameter model (b). 
(a)
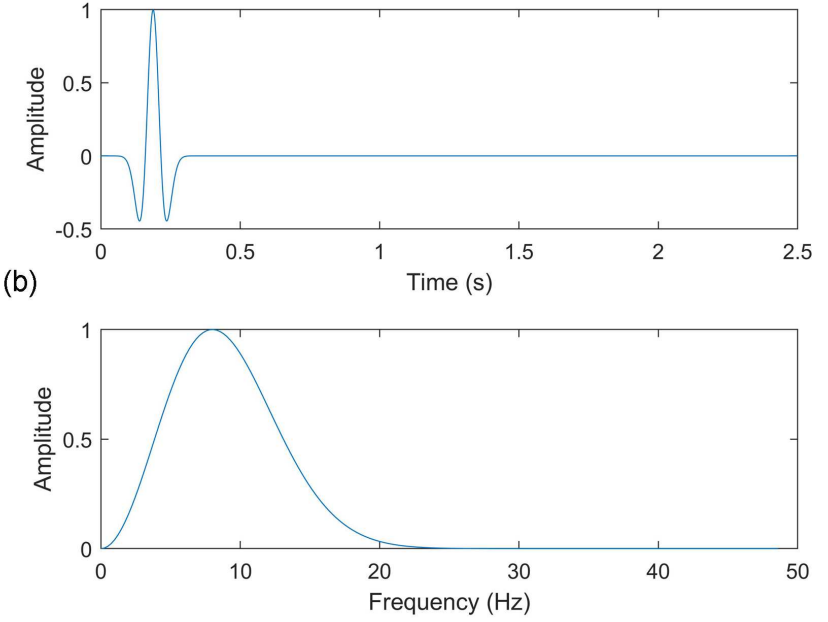

Figure 5: The Ricker wavelet (a), and the frequency spectrum corresponding to it (b). 

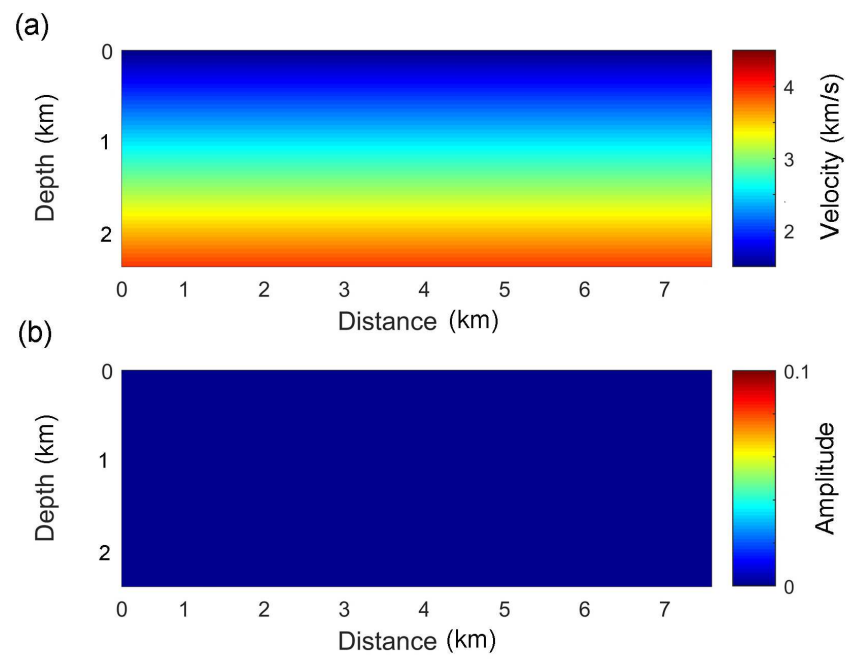

Figure 6: Initial NMO (horizontal) velocity $v_{n}$ (a), and anisotropic parameter model (b). 

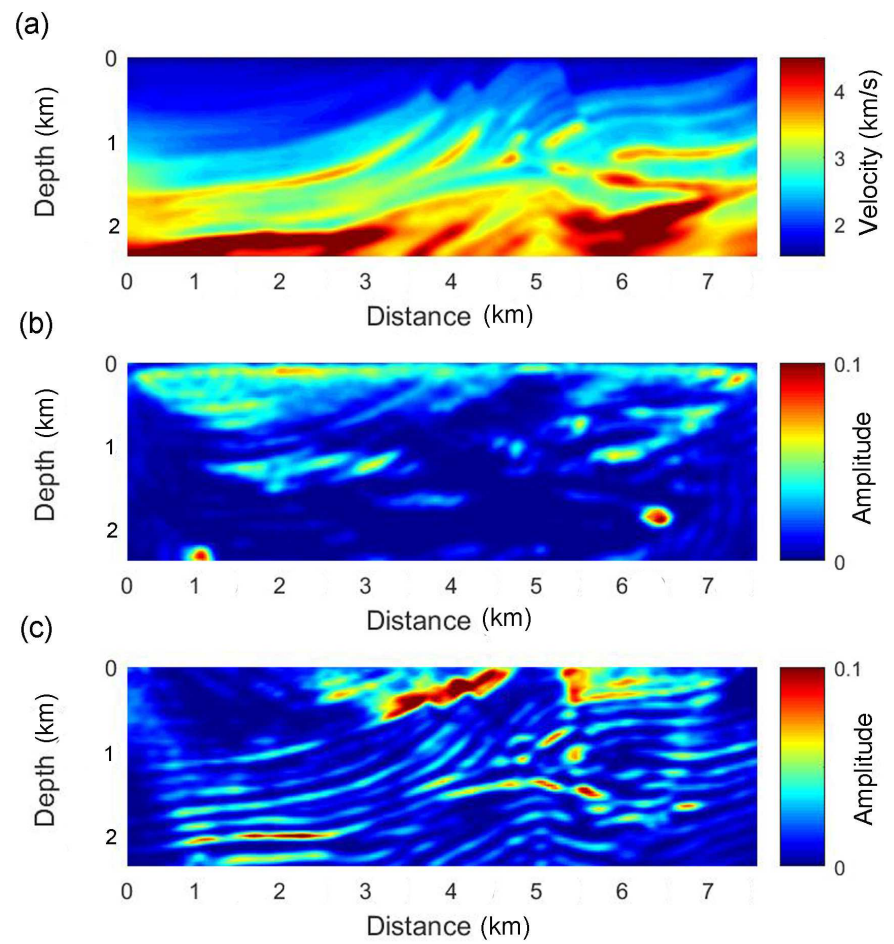

Figure 7: Inverted NMO velocity $v_{n}(\mathrm{a}), \eta(\mathrm{b})$ and $\delta(\mathrm{c})$. - 


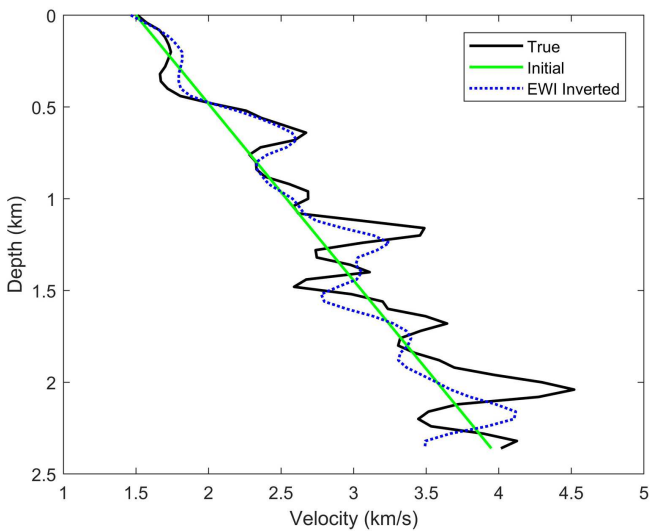

Figure 8: A vertical NMO velocity profile at $3.7 \mathrm{~km}$.- 


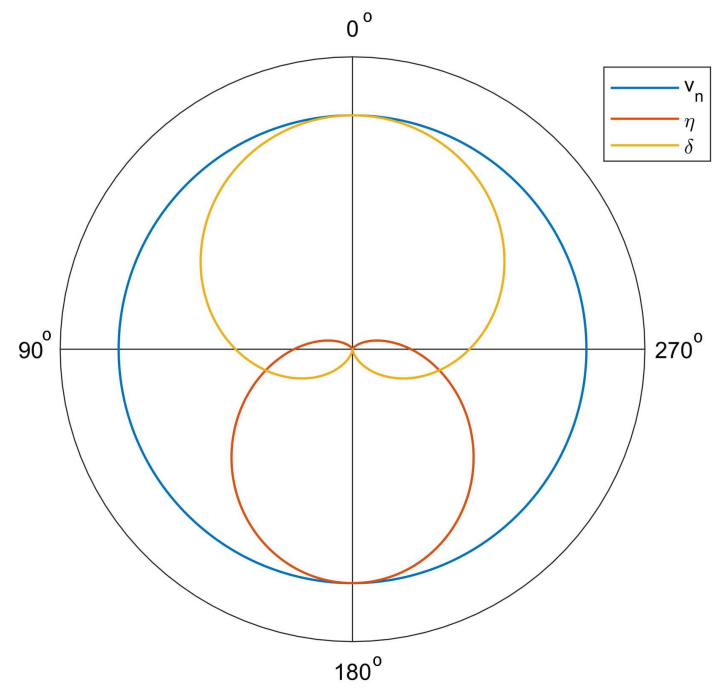

Figure 9: Radiation patterns for the parametrization $\left(v_{n}, \eta, \delta\right)$.- 

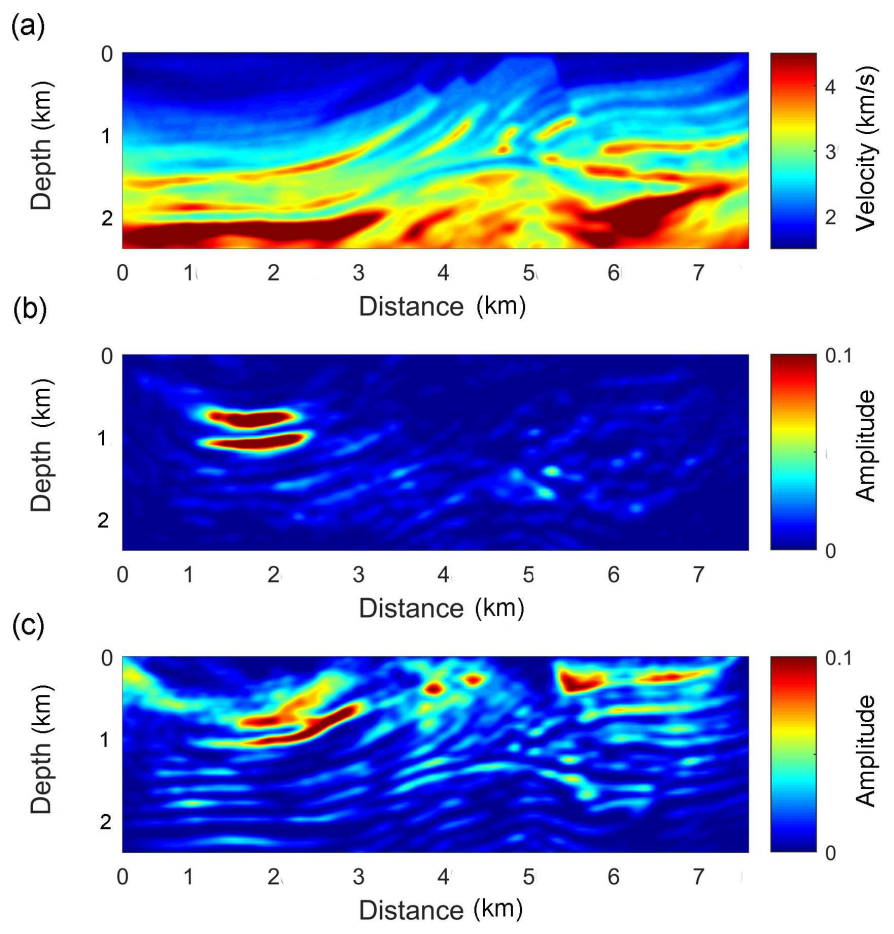

Figure 10: Inverted horizontal velocity $v_{h}(\mathrm{a}), \eta(\mathrm{b})$ and $\epsilon$ (c) using EWI. - 

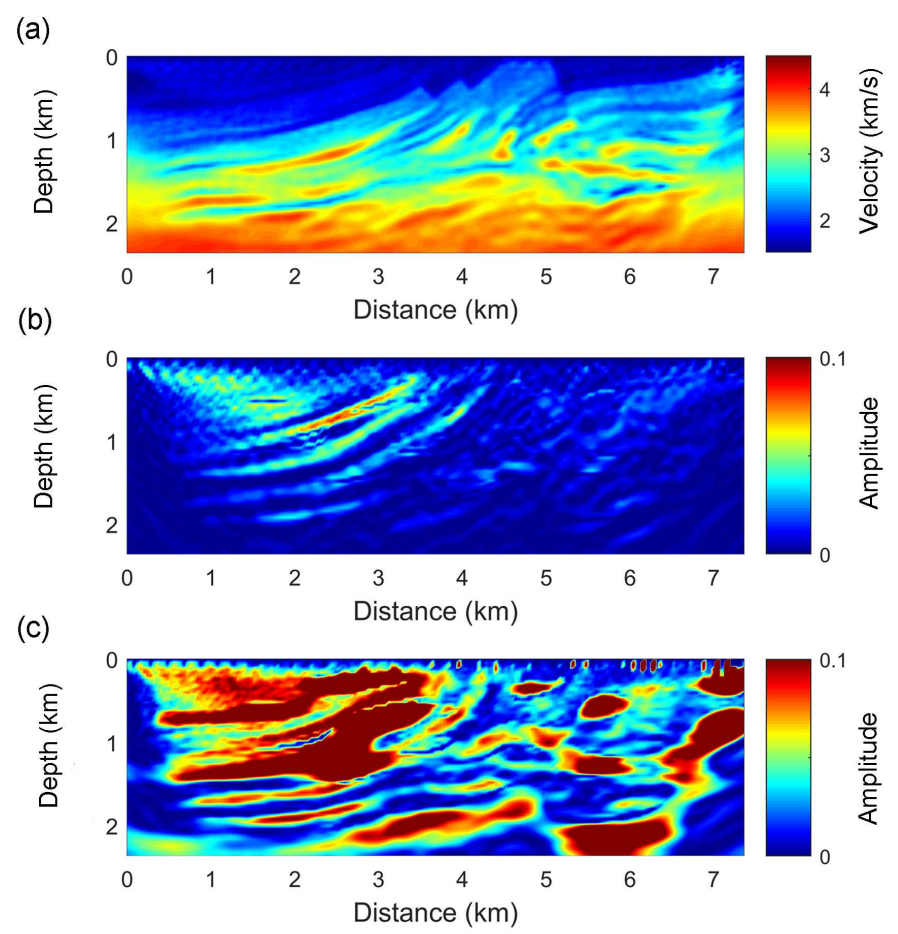

Figure 11: Inverted horizontal velocity $v_{h}(\mathrm{a}), \eta(\mathrm{b})$ and $\epsilon$ (c) using FWI. - 


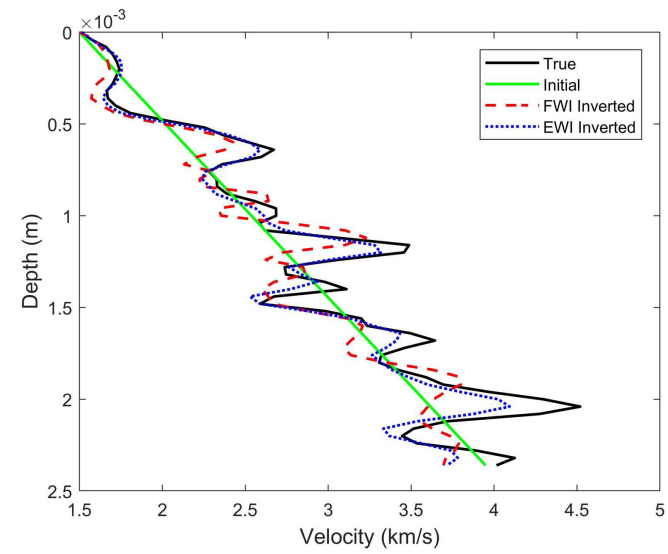

Figure 12: A vertical horizontal velocity profile at $3.7 \mathrm{~km}$.- 


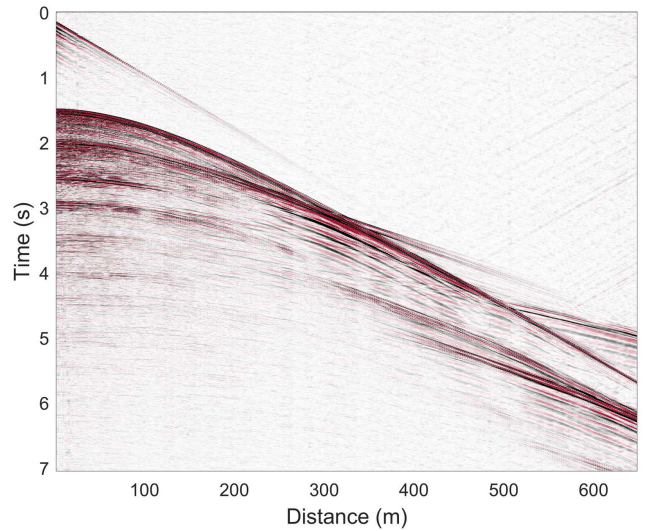

Figure 13: A shot gather from the real dataset. - 


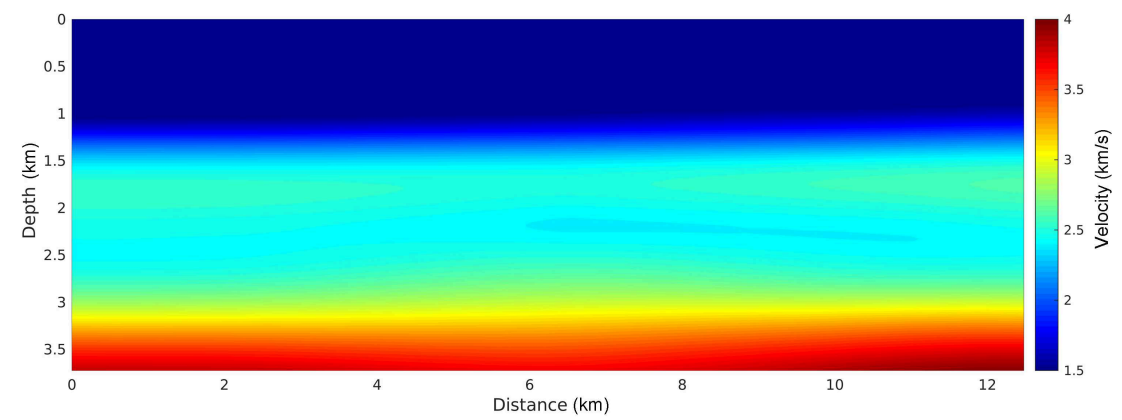

Figure 14: The initial velocity model. - 


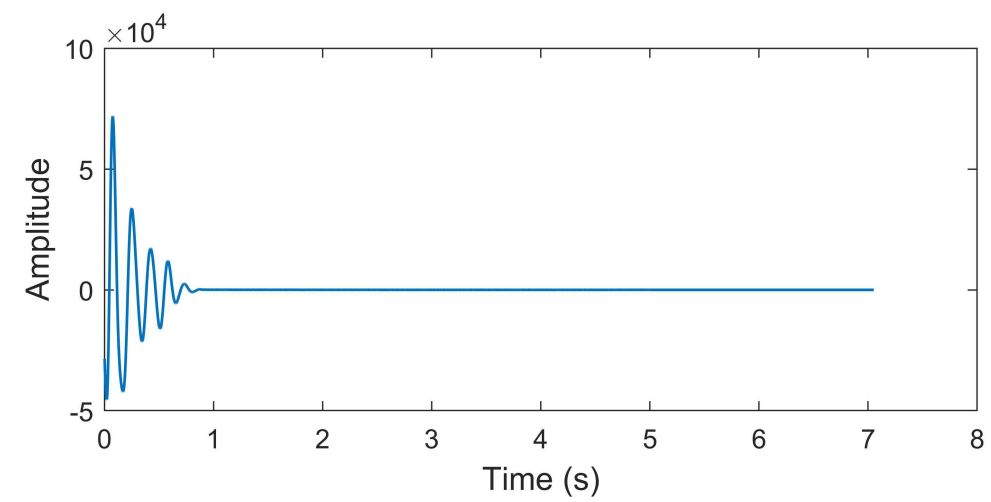

Figure 15: The estimated wavelet using the initial model. 


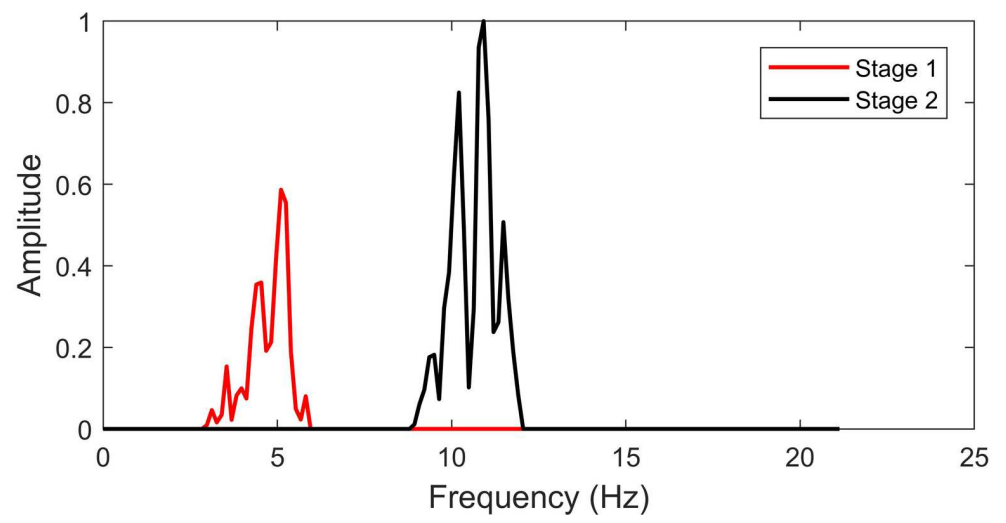

Figure 16: The frequency spectrum of the two selected frequency bands. - 


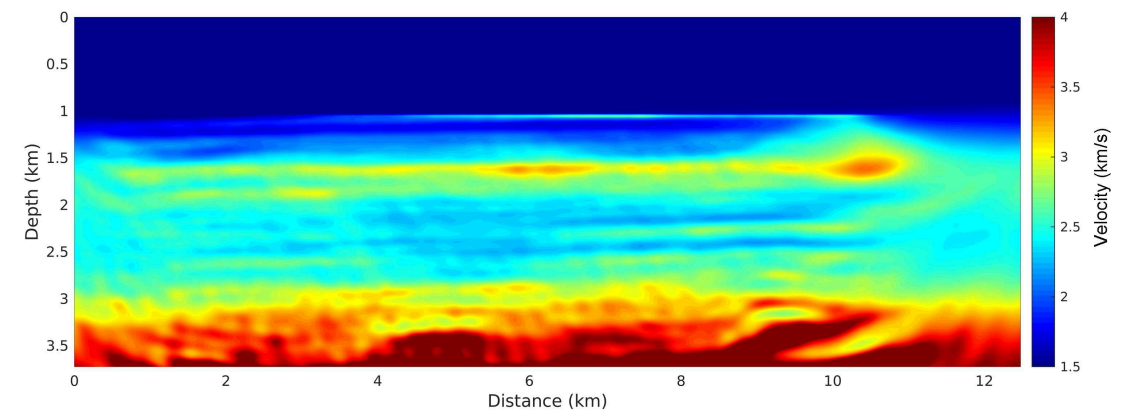

Figure 17: The inverted velocity using isotropic EWI. - 


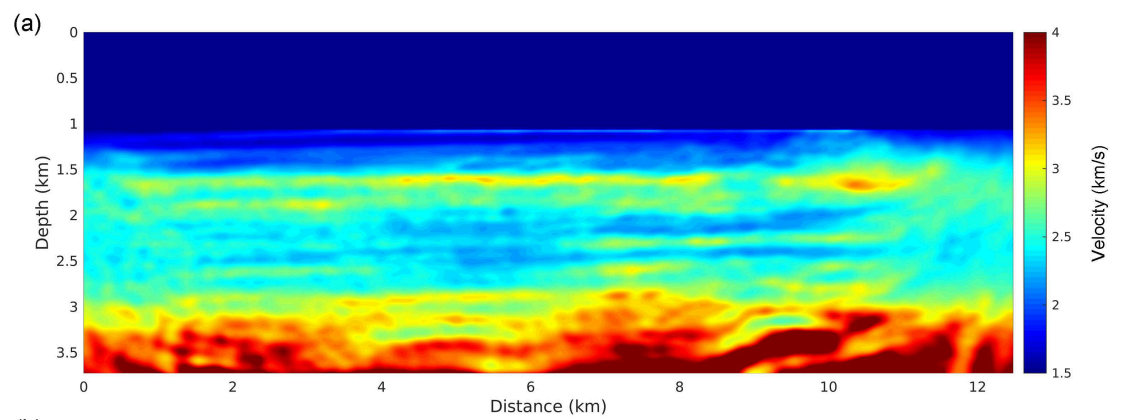

(b)
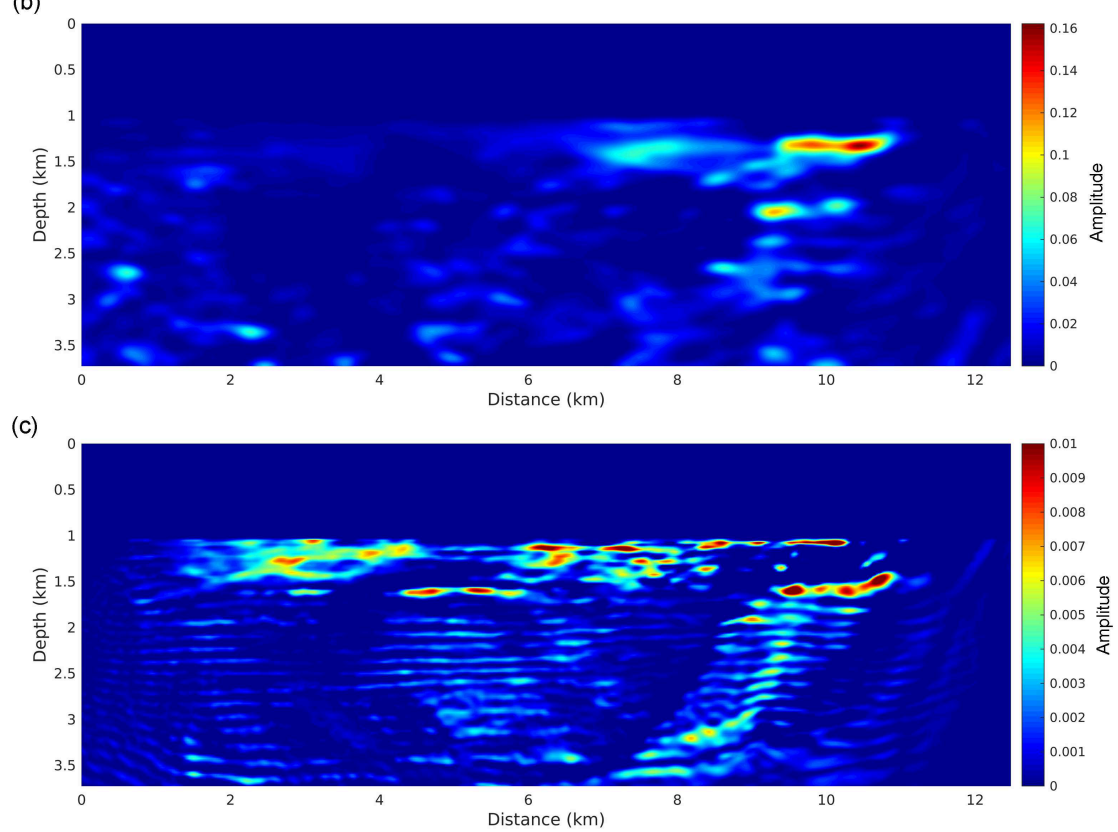

Figure 18: The horizontal velocity $v_{h}(\mathrm{a}), \eta(\mathrm{b})$, and $\epsilon(\mathrm{c})$. 


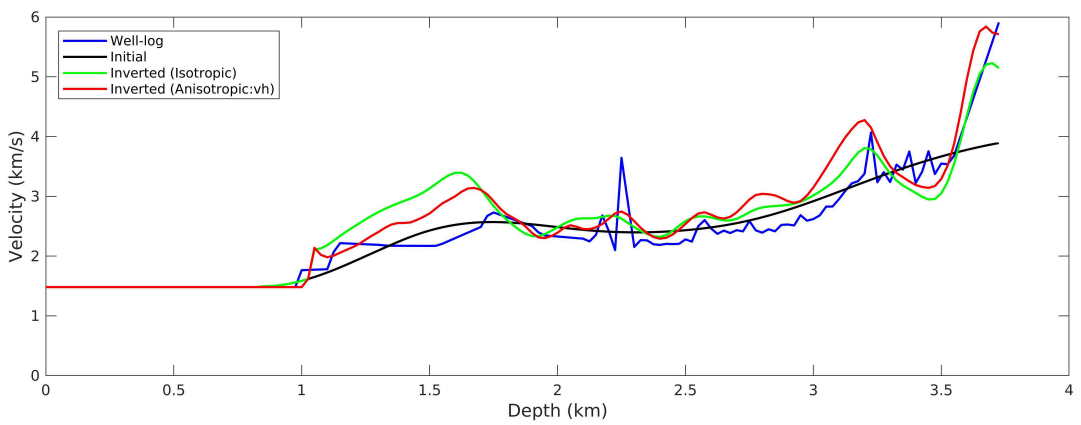

Figure 19: The horizontal velocity profile at $10.5 \mathrm{~km}$ compared to the well-log vertical velocity. 


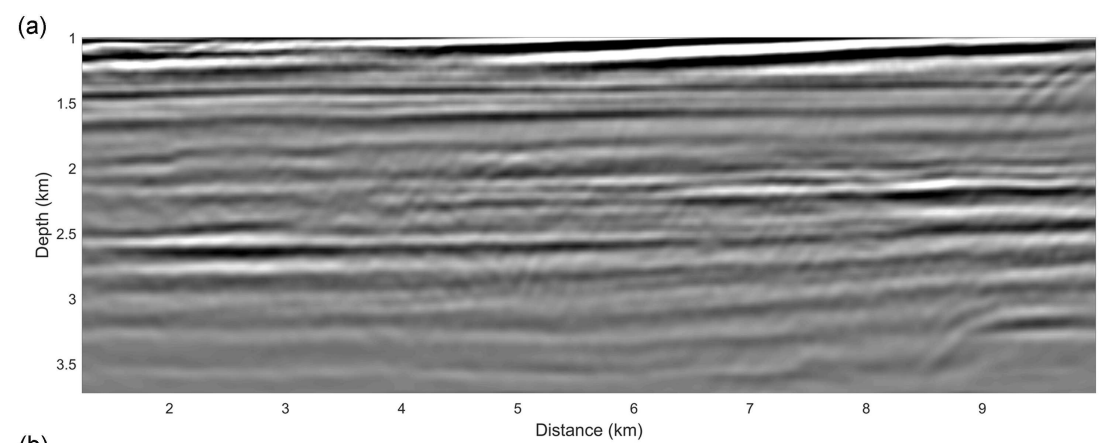

(b)

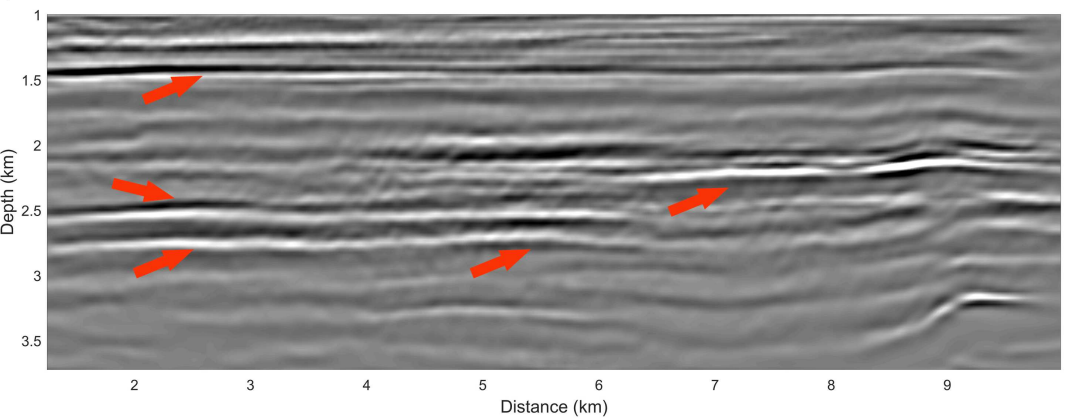

Figure 20: The RTM image for the initial (a) and inverted (b) models (the red arrows points to areas of clear improvements). 


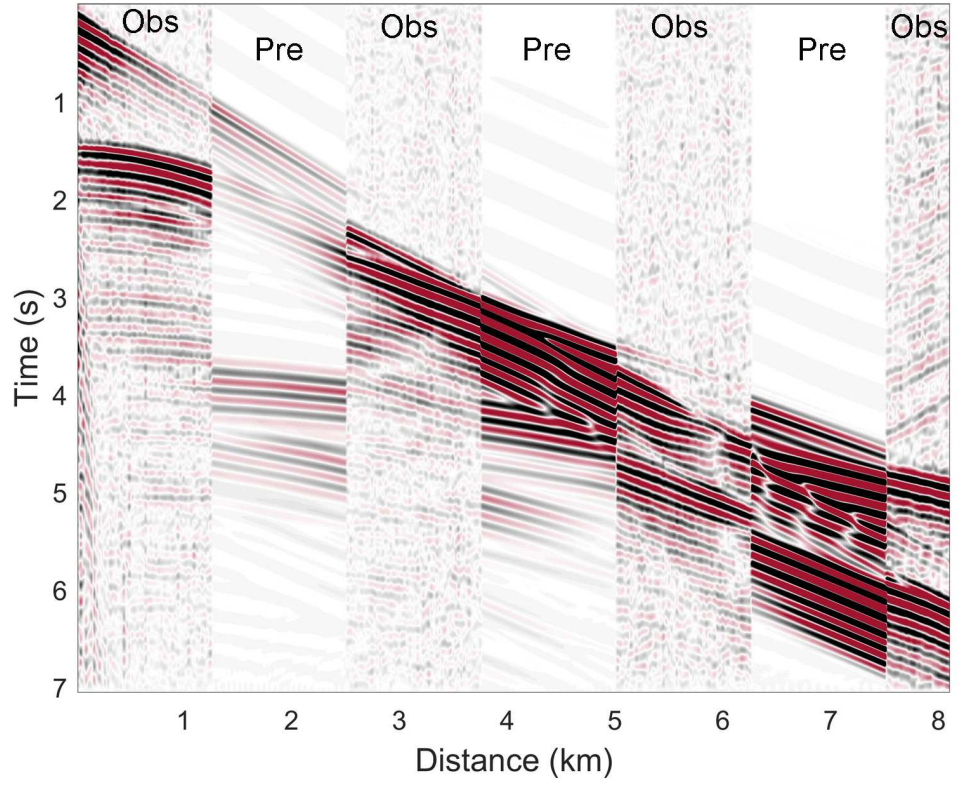

Figure 21: A shot gather displaying interleaved predicted (Pre) and observed (Obs) data using the initial model. 


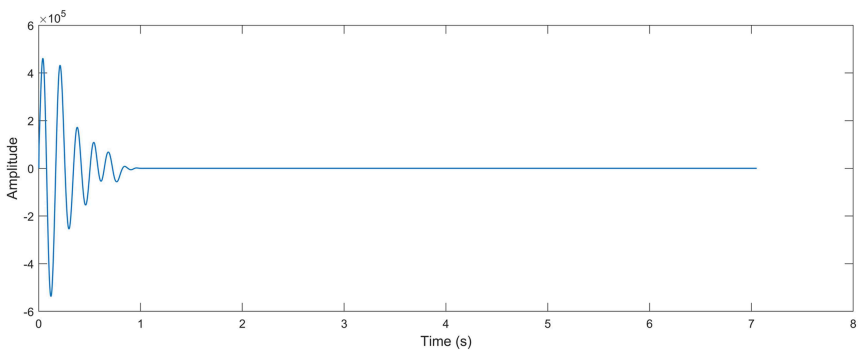

Figure 22: The estimated wavelet using inverted model. 


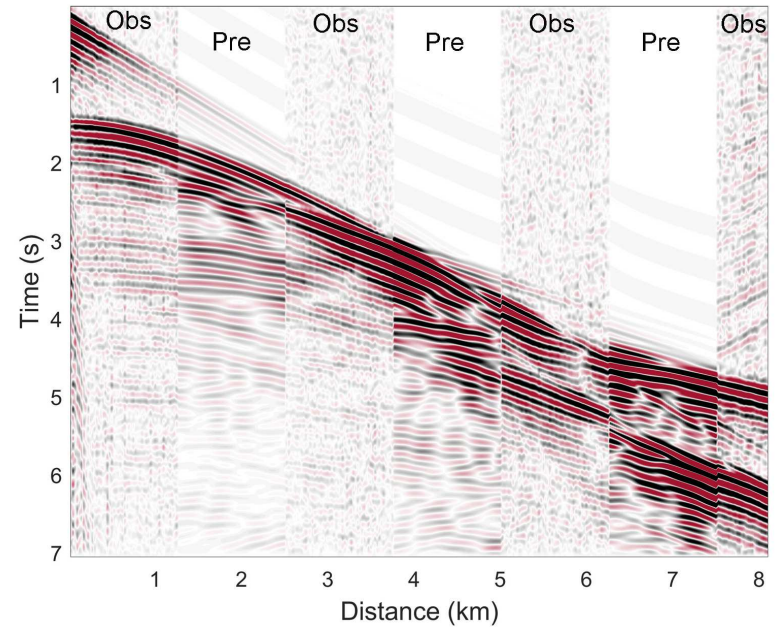

Figure 23: A shot gather displaying interleaved predicted (Pre) and observed (Obs) data using the inverted model. 